\title{
Use of human pluripotent stem cell-derived cells for neurodegenerative disease modeling and drug screening platform
}

\author{
Juan Antonio Garcia-Leon ${ }^{1,2}$, Javier Vitorica ${ }^{2,3,4}$ \& Antonia Gutierrez ${ }^{* 1,2}$ (iD) \\ ${ }^{1}$ Departamento Biologia Celular, Genetica y Fisiologia, Facultad de Ciencias, Instituto de Investigacion Biomedica de \\ Malaga-IBIMA, Universidad de Malaga, 29010 Malaga, Spain \\ ${ }^{2}$ Centro de Investigacion Biomedica en Red Sobre Enfermedades Neurodegenerativas (CIBERNED), 28031 Madrid, Spain \\ ${ }^{3}$ Departamento Bioquimica y Biologia Molecular, Facultad de Farmacia, Universidad de Sevilla, 41012 Sevilla, Spain \\ ${ }^{4}$ Instituto de Biomedicina de Sevilla (IBiS)-Hospital Universitario Virgen del Rocio/CSIC/Universidad de Sevilla, 41013 Sevilla, Spain \\ *Author for correspondence: +34 952133344; agutierrez@uma.es
}

Most neurodegenerative diseases are characterized by a complex and mostly still unresolved pathology. This fact, together with the lack of reliable disease models, has precluded the development of effective therapies counteracting the disease progression. The advent of human pluripotent stem cells has revolutionized the field allowing the generation of disease-relevant neural cell types that can be used for disease modeling, drug screening and, possibly, cell transplantation purposes. In this Review, we discuss the applications of human pluripotent stem cells, the development of efficient protocols for the derivation of the different neural cells and their applicability for robust in vitro disease modeling and drug screening platforms for most common neurodegenerative conditions.

First draft submitted: 18 October 2018; Accepted for publication: 13 February 2019; Published online: 4 June 2019

Keywords: Alzheimer's disease $\bullet$ amyotrophic lateral sclerosis $\bullet$ CRISPR-Cas9 • disease modeling • drug screening - hiPSCs • human-induced pluripotent stem cells • Huntington's disease $\bullet$ multiple sclerosis $\bullet$ neurodegenerative disease • Parkinson's disease

Neurodegenerative diseases are characterized by the chronic and progressive deterioration of neuronal functioning, resulting in cognitive impairment, memory deficits, deficiency in motor function, loss of sensitivity, affectation of autonomous brain system, changes in perception and/or mood, etc., with combination of these features present in individual patients. Several neurodegenerative diseases share common pathological pathways including abnormal accumulation of toxic-aggregated proteins, mitochondrial dysfunction, axonal transport defects and chronic inflammation, which ultimately lead to neurodegeneration [1].

Neurodegenerative conditions represent an enormous impact on global health due to their high incidence, the severity of their symptoms and, in general, the lack of effective therapies able to mitigate those symptoms and/or counteract the course of the disease [2]. In addition, the incidence of neurodegenerative diseases in developed countries is rapidly increasing, as is the case for Alzheimer's disease (AD) [3], amyotrophic lateral sclerosis (ALS) [4], multiple sclerosis (MS) [5], Parkinson's disease (PD) [6] and other forms of dementia as frontotemporal dementia, progressive supranuclear palsy or corticobasal degeneration [7]. Although the reasons for this general increase in the number of people affected by neurodegenerative diseases are not properly established, the general aging of the western-countries population and a higher exposure to certain substances as chemicals and a higher contamination, are few of the possible causes for this increase [4].

Modeling of neurodegenerative diseases has always been a difficult task, due to the complexity of the conditions, poor access to human samples and little knowledge of disease pathology. Animal models have been the most widely used, due to the intrinsic features of in vivo experimentation which allow, in principle, more accurate modeling. Nonetheless, disease modeling with animals requires the generation of reliable disease models, the use of a large number of animals and limits the combination of experimental conditions tested, due also to the long experimentation times required [8]. 
The advent of human pluripotent stem cell (hPSC) discovery and development has represented a new scenario complementing the modeling of neurodegenerative diseases. Since their discovery, important efforts have been established in the derivation of hPSC-derived neural cells from patients and healthy donors, which allow disease modeling using human samples with inherent advantages over in vivo models.

In this Review, we will discuss the current state of the art in neurodegenerative disease modeling and drug testing for the most prevalent neurodegenerative conditions, and provide data predicting the development of this fast-changing and promising field of research.

\section{Pluripotent stem cells}

Since the advent of human embryonic stem cells (hESCs) derivation in 1998 [9] and the discovery of murine and human-induced pluripotent stem cells (hiPSCs) in 2006-2007 [10,11], a new field of research was opened up. Although stem cells have represented a promising strategy for their potential therapeutic uses, which have already been investigated for more than two decades, successful research performed in the past few years have revealed a prominent utility of stem cells for disease modeling and drug screening purposes [12].

Human ESCs and iPSCs are pluripotent stem cells, with hESCs deriving from the inner mass of the blastocyst. On the other hand, hiPSCs are generated by the forced expression of reprogramming factors, with the transcription factors OCT3/4, SOX2, KLF4 and c-MYC constituting the initial reprogramming cocktail [11]. Both hESCs and hiPSCs have the capacity, under certain defined conditions, to differentiate toward any somatic cell type, as will be expanded later.

The employment of hPSCs for disease modeling was initially delayed due to the relatively poor yield of initial methods for their derivation, complexity and costs. Nonetheless, several improvements in methodology for their generation have been developed. Among these improvements, combination of different reprogramming factors [13] and the combined use of different compounds [14] have contributed to increase the efficiency of reprogramming in a great extent. In addition, methods to introduce reprogramming factors relied in the use of integrating viral vectors as retrovirus and lentivirus due to their efficiency, although this technology precludes a potential further use of these cells and leads to random integration events hindering the standardization of this approach among labs. Recently, other methods for transgene delivery have been developed including the delivery of reprogramming factors by means of targeting the cells with episomal vectors, non-integrating Sendai virus and the use of mRNAs. These technologies lead to the generation of iPSCs with similar efficiencies that those derived using viral vectors and allow the generation of GMP-grade iPSCs compatible with their use in the clinical setting [15].

These advances have allowed the extension in the use of hPSCs among labs for disease modeling and drug screening purposes (Figure 1). This has represented a considerable advance in the field of neurodegenerative conditions that, until the implementation of hPSC technology, had been modeled exclusively and only partially by the use of animal models without full recreation of disease phenotype [16]. hPSC technology has transformed the modeling of neurodegenerative diseases, since it has allowed the study from familial monoallelic to complex multifactorial diseases in the context of patient-derived neural cells and tissue, systems that previously were not available for experimental investigation. The ability of hPSCs to differentiate toward any somatic cell type allows the generation of neural cells implicated in the pathogenesis of neurological diseases, where insertion or correction of disease-associated genetic mutations can be performed.

\section{Gene-editing in hPSCs}

An important and sometimes poorly considered challenge of hPSC technology is the variability between lines from the same individual or from the same group of individuals in terms of their capacity to differentiate toward different lineages. These differences are due to intrinsic genetic backgrounds, differences in reprogramming methods and/or epigenetic status of the somatic cell at the time of reprogramming [17]. Therefore, the generation of isogenic pair cell lines by correction or introduction of desired disease-associated genetic modification $(s)$ is the best approach for assessing the biological effects exerted by one or several disease-associated mutations. Making such isogenic control lines requires the use of site-specific nucleases targeting the desired location of the genome.

The use of site-specific nucleases to generate double-strand breaks in the genome allowed an efficient modification of the genetic sequence [18]. The complexity of the initial technologies and their scarce customization limited the wide implementation of this technology [19]. An important revolution in the field came by the employment of DNA-binding zinc finger nucleases for genomic editing, which notably increased the specificity and efficiency of the approaches, especially when cells were provided with a 'donor plasmid' (an exogenous DNA flanking the 


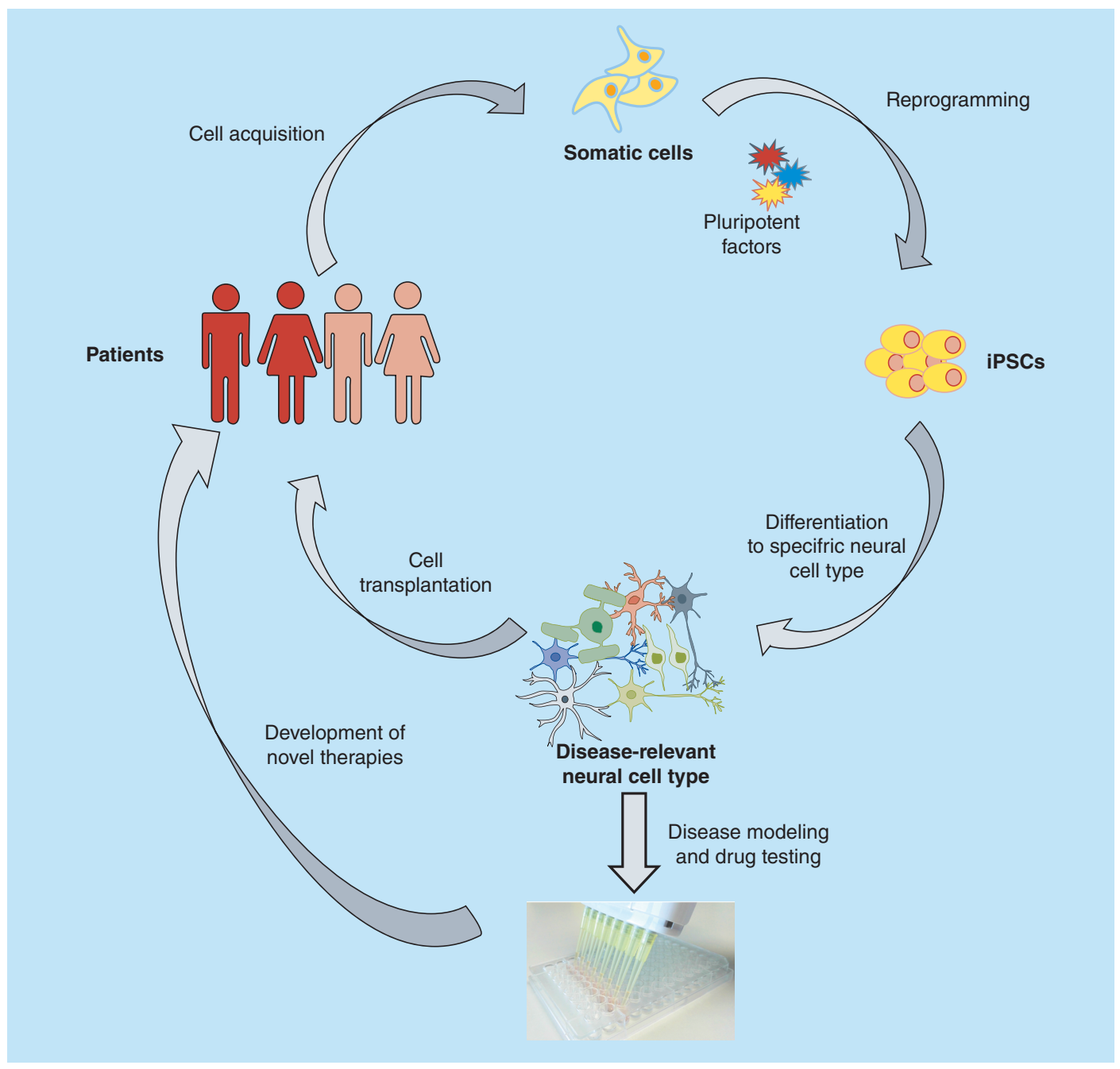

Figure 1. Schematic representation of the potential applications of induced pluripotent stem cells obtained from patients with neurodegenerative diseases. Human iPSCs can be differentiated toward disease-relevant neural cell types to be used potentially directly for cell transplantation or to be used as a platform for disease modeling and drug screening, which can lead to the development of novel and effective therapies.

iPSC: Induced pluripotent stem cell.

cutting site and with great homology with the target locus), which can be used by the cell as a template to repair the double-strand brake by homology recombination [20], a technology which can be applied to the genomic editing of hESC and hiPSCs as well [21].

In 2010, Christian et al. used a novel system by fusing transcription activator-like effectors (TALEs) to the catalytic domain of the Fok I endonuclease to generate TALE nucleases (TALENs) [22]. TALENs are easier to customize and less restrictive about the requirements the sequence must compile to be targeted. Moreover, this system showed a higher efficiency and accuracy over previous technologies [23].

However, the real revolution in the field came with the discovery and adaptation for genome editing of the CRISPR/CRISPR-associated (Cas) adaptive immune systems [24], with the variant CRISPR-Cas9 being the most widely used [25]. This complex is formed by the Cas9 nuclease associated with a base-paired structure formed between the activating tracrRNA and the targeting crRNA to cleave the target double-strand DNA. Site-specific cleavage occurs at places determined by both base-pairing complementarity between the CRISPR RNA (crRNA) and the target protospacer DNA and a short motif (the protospacer adjacent motif), next to the complementary region in the target DNA. As result, the Cas9 endonuclease can be programmed with single RNA molecules to 
cleave specific DNA sites, leading to double-strand breaks in the genome that can be used for introducing desired modifications by co-providing a donor template DNA with desired modifications [24].

The advent of having a customizable RNA sequence (in contrast with protein sequence modifications needed for customization of zinc finger nucleases and TALENs) has resulted in a powerful gene-editing tool which, in addition, is cheaper and easier to design and implement than previous technologies. These features have made CRISPR-Cas9 system a widely used tool for gene-editing. Immediately after their discovery, they have been applied for performing gene-editing of hPSCs [26]. hPSCs are characterized by a poor efficiency of transfection, so specific gene-editing strategies should be followed to generate efficient modifications in the genome of these cells [27]. In this sense, the incorporation of selectable cassettes in safe harbor loci in the genome [28] or a gene-editing strategy including the incorporation of selectable cassettes with a subsequent removal in a seamless manner [29] allow a more efficient targeting strategy.

CRISPR-Cas9-mediated gene editing is an excellent tool for modeling some monogenic diseases or to study the contribution of single or several gene variants to a certain pathology. In this sense, hiPSCs derived from patients carrying a certain mutation associated with a neurodegenerative disorder can be used not only to model the disease but through the correction of this specific mutation and generation of isogenic cell lines, the contribution of a certain genetic modification to the disease phenotype can be addressed in a human model. In the case of familial ALS cases with mutations in the FUS gene, Guo et al. using iPSC-derived motor neurons (MNs) from these patients and correcting the mutations using CRISPR-Cas9 technology demonstrated that the presence of FUS mutations were responsible for shortcomings in axonal transport, a defect which could be counteracted by pharmacological inhibition of the HDAC6 gene as well, identifying a possible therapeutic target for the disease [30]. In the case of AD, studies employing the CRISPR-Cas9-mediated gene targeting in iPSCs are emerging, elucidating the pathogenic role of disease-associated mutations as those present in PSEN2 [31], PSEN1 [32], APP [33] or APOE [34]. Moreover, CRISPR-Cas9-mediated gene editing has promised utility in the treatment of neurodegenerative diseases, as it has been shown for PD [35]. On the other hand, CRISPR-Cas9 can be used for the introduction of pathogenic variants for disease modeling. In this sense, we have recently generated a tauopathy model by introducing three MAPT mutations using CRISPR-Cas9 combined with the piggybac transposase in a seamless manner in iPSCs, leading to the manifestation of neurodegenerative phenotypes in iPSC-derived cortical neurons [36]. Therefore, gene-editing strategies have evolved in parallel with the use of hPSCs for disease modeling, a fact that has just started to prove fruitful for the combination of these two tools for the discovery and validation of novel therapeutic strategies [37].

Despite the potential of the combination of gene-editing in hPSCs for disease modeling and as a platform for drug testing, there are several drawbacks that limit this technology. One of these limitations deals with the fact that most neurodegenerative diseases are caused not by monogenic mendelian mutations but are consequence of environmental factors together with multiple low-effect-size-risk alleles identified in genome-wide association studies. This makes modeling with hPSCs a difficult task since disease-associated gene variants differ among patients and are not a causative agent per se to develop the disease or the pathogenic phenotype. To circumvent this issue, one possible strategy would be to generate several iPSC lines from patients with different genetic backgrounds in combination with multiplexed CRISPR-Cas9-mediate gene-editing to address the implication of those variants and/or discover novel disease-driving molecular pathways [38].

Another limitation of gene-editing strategies (with special focus on CRISPR-Cas9) deals with nuclease specificity and off-target events. Several studies have determined the rate of off-target events produced by site-specific nucleases, focusing on the CRISPR-Cas9 system. Initial studies determined that the possibility of having off-target effects in a targeted cell or organism was $\geq 50 \%$ [39], limiting the use of this technology. Nonetheless, more accurate methods for measuring those off-target events [40] and the last optimizations of the CRISPR-Cas9 technology have reduced practically to undetectable the frequency of off-target mutations [41]. However, this phenomenon should always be taken into account for the experimentation performed.

\section{Methods for the derivation of neural cells from hPSCs}

One of the intrinsic features of pluripotent stem cells is that, when exposed to defined culture conditions, are able to differentiate toward (almost) any somatic cell type [42]. Based on this, soon after the derivation of hESCs, and especially after the discovery of hiPSCs, researchers have made important efforts to elucidate molecular pathways governing cell differentiation toward the different lineages, with a special focus on the derivation of neural cells, since access to primary brain tissue is scarce and, when available, expansion of the different cell types ex vivo is 


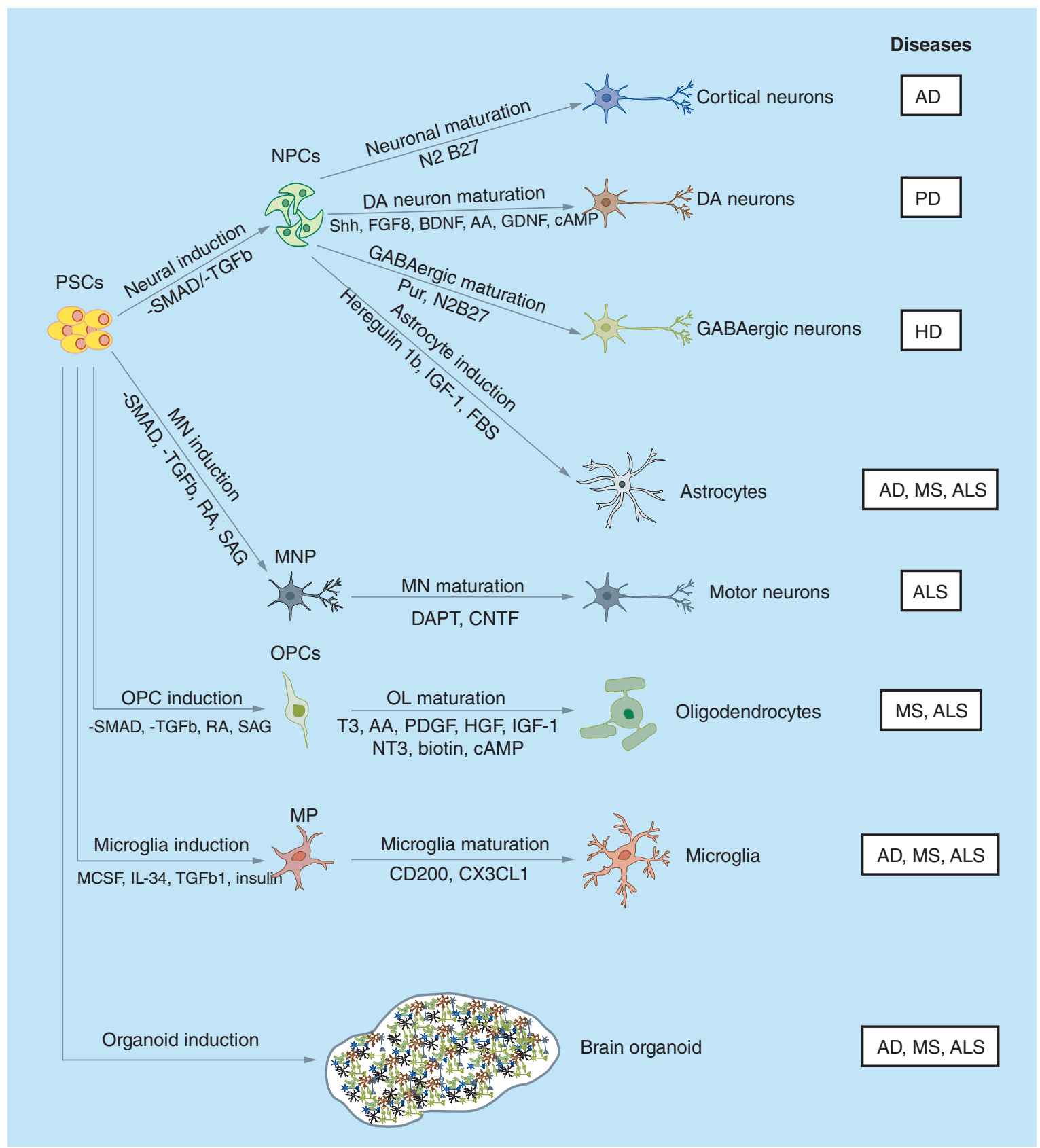

Figure 2. Layout of culture conditions for the derivation of human pluripotent stem cells into the different neural cell types and relevance of each cell type to neurodegenerative diseases.

AD: Alzheimer's disease; ALS: Amyotrophic lateral sclerosis; HD: Huntington's disease; MS: Multiple sclerosis; PD: Parkinson's disease.

very limited. Next, we are going to discuss the progress made for the optimization of the differentiation methods developed to generate the different neural cell types from hPSCs (see Figure 2).

\section{Induction of a neural fate \& neuronal differentiation}

Soon after hESCs derivation, different attempts were made to fate pluripotent stem cells toward a neural phenotype. It was observed that when hPSCs are cultured in standard conditions without pluripotency-sustaining factors, neural fate is spontaneous induced, with the TGF $\beta$-related signaling negatively inducing this differentiation [43]. Later on, using specific TGF $\beta$ antagonists to abolish the SMAD signaling, Chambers et al. improved and defined 
the differentiation method obtaining a robust and efficient neural conversion from both hESCs and hiPSCs [44]. Nonetheless, these derivation methods did not recreate human neurogenesis in a temporal manner, with important limitations for disease modeling. In 2012, Shi et al. circumvented these issues since they developed an improved method for neuronal specification based on known rodent cortical development that included, after neural progenitor specification based on TGF $\beta$-signaling inhibitors, an extended period of cortical neurogenesis and terminal neuronal maturation, which recreate human corticogenesis with the generation of deep and upper layer cortical neurons in a specified temporal manner [45]. This methodology enables studies to unravel the development of human cerebral cortex and the generation of individual-specific neurons that could be used for disease modeling and therapeutic approaches. Further improvements in the methodology to obtain electrophysiological active neurons have been described [46], also using defined and standard culture conditions without the need of co-culturing the generated neurons with exogenous cells [47].

In addition, protocols for generating specific neuronal subtypes have been established. In this sense, specific protocols have been developed to generate dopaminergic (DA) neurons from hPSCs. Nguyen et al. achieved efficient generation of DA neurons by a protocol performing neural induction by dual-SMAD inhibition, followed by culturing the cells in specific DA neurons cues including Sonic hedgehog and FGF8b signaling, supporting the cells with additional growth factors as BDNF, ascorbic acid, GDNF and cAMP; obtaining DA neurons able to reproduce key PD features in vitro [48]. Further optimizations of the protocol have been performed, leading to a more efficient generation of DA neurons able to engraft murine and non-human primates [49-51].

MNs have been another neuronal population widely studied due to their implication in several neurodegenerative conditions as ALS, where selective MN death occurs [52]. Since the discovery of hiPSCs, several protocols have been developed for the specific differentiation of these cells into functional MNs [53-56]. Maury et al. tested several small molecules targeting developmental cues for the derivation of different neuronal subtypes [57]. They obtained an improved method for MN generation consisting of neural specification induced by SMAD inhibition and Wnt pathway activation, followed by caudalization and ventralization induced by retinoic acid and smoothened agonist, respectively; culturing the cells in the presence of the gamma secretase inhibitor $\mathrm{N}$-[N-(3,5-Difluorophenacetyl)L-alanyl]-S-phenylglycine t-butyl ester (DAPT) and ciliary neurotrophic factor [30]. Although cell-replacement therapies are far from being achieved due to the complex and still unsolved pathology of ALS, hPSC-derived MNs can be used to resolve the complex pathology and serve as a platform to test altered pathways [37].

Apart from DA or MNs, other neuronal subtypes have been generated by finely exposing pluripotent cells to the signals governing telencephalic development, in a time and concentration-dependent manner [58].

\section{Generation of astrocytes from hPSCs}

Astrocytes are the most common glial cells present in the CNS, with essential functions in neuronal metabolic support, synapse formation and regulation of blood flow, among others [59]. Astrocytes are a heterogeneous cell type emerging from neural precursors in common with neurons that results in mixed generation of neurons and astrocytes in several protocols of hPSC differentiation [47]. Nonetheless, specific methods have been developed for efficient generation of functional astrocytes from hPSCs in defined culture conditions that resemble primary astrocytes and maintain their functionality after in vivo transplantation [60]. Further optimization in the protocols has been performed, obtaining more efficient methods to generate astrocytes able to unleash immune responses and able to support phagocytosis mediated by microglial cells [61]. hPSC-derived astrocytes can be generated from individuals suffering from different neurodegenerative diseases and can reproduce several phenotypes encountered in vivo, a feature that can be employed for effective disease modeling and the discovery of new therapeutic targets [62-64].

\section{Generation of oligodendrocytes from hPSCs}

Since the successful isolation and culture of hESCs, different groups developed specific protocols for the generation of oligodendrocyte precursor cells (OPCs) and oligodendrocytes (OLs), which are the cells responsible for myelin synthesis in the CNS. OLs and OPCs are a heterogeneous population of cells existing at different maturation states that, apart from being the cells responsible for myelination, have important trophic and metabolic functions [65]. Nistor et al. in 2005 was the first to describe the generation of OLs from hPSCs through neurosphere formation after a 10-15 week protocol, using only one hESC line [66]. Further improvements in the methodology were made, but all these protocols remained inefficient (very long differentiation times and low yield) and their robustness was in debate due to reduce number of cell lines tested $[67,68]$. All these protocols attempted to recapitulate the different processes and signals that occur during development. However, the different protocols differed in the combinations 
of growth factors, cytokines and other molecules needed, which highlight that OL specification in vivo is achieved in different developmental regions that respond to specific local concentration of different factors [69]. Since the discovery of iPSCs, several groups developed specific protocols to obtain OPC/OLs from hiPSCs. Initial studies adapted previous protocols developed for hESCs to hiPSCs [70,71]. In 2013, Wang et al. developed an optimized protocol for OPC/OL generation from hiPSCs, accounting for a total differentiation time of 150 days, time after which they obtained $70-80 \%$ of OPCs, with a small fraction of those differentiating toward mature $\mathrm{MBP}^{+}$OLs able to engraft and myelinate the myelin-deficient shiverer mice [72]. Douvaras et al. in 2014 published an improved version of the protocol, consisting in a first stage generating OLIG2+ OPCs in suspension, which were dissociated and plated to mature till day 75-90, when $\mathrm{MBP}^{+}$mature OLs started to appear [73]. Generated OPCs/OLs using this protocol were able to myelinate neurons in vivo and could be generated from MS patient lines. In line with this, Piao et al. showed the clinical applicability of hPSC derived-OPCs that were able to remyelinate and rescue an irradiated rat model of brain tumor radiation [74].

Although the last protocols reduced the required time needed for OPC/OL generation and increased the efficiency, the generation of OLs from hPSCs remained inefficient and required long differentiation times in culture, limiting the use of these cells. To circumvent these issues, we and others have developed specific protocols that notably increase the efficiency of OL generation and reduced the differentiation time [75,76]. We demonstrated that forced expression of SOX1O alone leads to efficient OL generation within 20 days from hPSC stage, with generated OLs able to myelinate neurons both in in vivo and in vitro contexts. Moreover, hPSC-derived neuron-OL cocultures can be used for addressing the effect on myelin production of myelinating drugs in a high-throughput manner, serving this system as a platform where to test and/or validate the effects of candidate drugs [76].

\section{Generation of microglia from hPSCs}

The developmental origin of microglia has recently been elucidated, with few studies demonstrating that microglia arise from early yolk sac-derived hematopoietic progenitors, which migrate to the brain early in the development [77]. This fact has delayed the development of specific protocols to generate microglial cells from hPSCs. Several protocols were developed generating CD14 ${ }^{+}$monocytes/macrophages from hPSCs [78,79]. These cells express phagocytic markers, are morphologically similar to blood-derived monocytes and macrophages, produce cytokines in a similar fashion as macrophages depending on the activation status of the cell and display high phagocytic capacity [80]. Other protocols tried to generate specifically microglial cells, but these protocols failed to resemble bona fide microglia since they did not recapitulate precisely microglial ontogeny $[81,82]$.

In the past 2 years, a number of different protocols for generating microglial-like cells from hPSCs have been developed [83-86]. Although the methodology differed among the different approaches developed, these protocols have in common a first phase where early mesodermal progenitors are induced from hPSCs, followed by a maturation state where microglial-specific signaling pathways as IL34 and TGFb are induced [83-86]. This results in the generation of microglial-like cells resembling primary microglia at the transcriptome level, able to response to immune inducers and able to phagocytose amyloid- $\beta$, serving these cells as a platform for disease modeling and possibly drug testing [86].

\section{Brain organoids}

Much of our current understanding of human brain development and functioning relied on experimental studies performed mostly on mouse models, which do not fully mimic the features present in the human brain. Threedimentional CNS organoids possess the unique advantage of recapitulating multiregional and region-specific cytoarchitecture seen in the early human fetal brain development, becoming a strong complement for studying brain development and pathology, to develop new therapies for treating neurodevelopmental diseases [87]. In the past few years, different groups have reported the generation of human brain organoids from PSCs. Lancaster et al. were the first to demonstrate that brain organoids from hPSCs can be generated that recapitulate the human brain development generating different discrete but connected brain areas defined by the expression of specific cell markers and transcription factors, which can be used as well for modeling microcephaly [88]. After this discovery, other groups have used this approach to generate brain organoids that have resulted useful for modeling different developmental diseases as autism spectrum disorders [89], schizophrenia [90], microcephaly induced by Zika virus infection [91] and others [87]. In addition, 3D culture systems have been proved useful for modeling neurodegenerative diseases as $\mathrm{AD}[92,93]$ and dys-/demyelinating diseases [94]. 


\section{Use of hPSC-derived cells for drug screening \& validation platform}

For decades, drug discovery and validation relied on experimental testing of a certain number of compounds in animals to observe the effects produced on phenotypes, processes that were costly and time-consuming, allowing the test of a limited number of compounds. Advances made in chemical synthesis and automation platforms altered the paradigm of drug discovery, allowing the development of high-throughput screenings (HTS). HTS allow testing of hundreds or even thousands of compounds in a short period of time and with relative low costs. Classical HTS have been based on simple platforms where the effect of candidate compounds were tested on purified proteins, RNA, DNA or a simple mixture of a few molecules [95]. More complex platforms relied on the use of simple organisms (as yeasts) or cell lines that allow to study the effect of candidate drugs on alive organisms [96]. Although efficient, these assays usually present a major problem that is the poor validation rates of initially identified hits in in vivo models reproducing the disease, due mostly to the scarce biological relevance of the screening platforms related to the disease to treat and the use of non-human derivatives, which do not hold specific human biology. This problem may be circumvented by the use of more reliable models as screening platforms. In this sense, hPSCs may represent an excellent system where to perform drug validation and even being used for drug discovery. Although inefficient protocols for the derivation of specific cell types have precluded the use of this technology for drug testing [65], we believe recent literature indicates that it is the right moment to perform this technology translation, and several small biotech companies as well as big pharma industries are developing stem cell-derived platforms for drug testing.

Certainly, the use of hPSCs for drug testing has been earlier implemented in the case of hPSC-derived hepatocytes [97] and cardiomyocytes [98], possibly because the phenotypes to be observed after drug treatment are robust and easy to evaluate. For neurodegenerative diseases, this implementation has been slower due mostly to the inherent complex disease pathology, the inefficient protocols for the generation of neural cells and the lack of clear phenotypes to be evaluated. Next, we are going to discuss recent advances in disease modeling and drug screening for the main neurodegenerative diseases focusing in the employment of hiPSCs.

\section{Alzheimer's disease}

$\mathrm{AD}$ is the most prevalent neurodegenerative disease and form of dementia, characterized by the accumulation of amyloid-beta $(\mathrm{Ab})$ plaques and TAU-laden neurofibrillary tangles, with huge impact on society [99]. The pathology of $\mathrm{AD}$ is very complex and still not fully understood, with a recently described key role for microglial dysfunction in the disease [100-102]. Due to this fact and because it is a noncell autonomous disease, the disease modeling with hPSC-derived cells in AD has been hindered.

Most of the studies performed have focus on reproducing the Ab pathology present in $\mathrm{AD}$ (aberrant extracellular accumulation of the Ab42 peptide). Several groups have demonstrated that neurons generated from iPSCs of AD patients show an increased secretion of Ab42 [103] and this platform can be exploited for testing of drugs diminishing this phenomenon. Yahata et al. in 2011 generated hiPSC-derived cortical neurons, able to produce Ab40 and 42 peptides. They inhibited the function of beta and gamma secretases (enzymes implicated in the synthesis of Ab) by employing beta- and gamma-secretase drug inhibitors as well as a nonsteroidal anti-inflammatory drug, finding that those drugs reduced Ab40-42 content [104]. Using a similar platform, Xu et al. tested over 100 drugs, finding that a Cdk2 inhibitor was able to reduce Ab toxicity [105]. Nonetheless, drugs targeting Ab production pathways have resulted inefficient in clinical trials [106], evidencing a more complex disease pathology. In this sense, Mertens et al. found that iPSC-derived neurons do not respond to clinical doses of nonsteroidal anti-inflammatory drug-based gamma-secretase modulation [107]. Kondo et al. established an iPSC-derived neuronal drug screening platform where they tested over 1000 compounds for their ability of reducing Ab load within the culture. They obtained 27 hits that resulted in six leading compounds and, to maximize the anti-Ab effect, a combination of three compounds (bromocriptine, cromolyn and topiramate) was selected as anti-Ab cocktail, which showed efficacy in reducing $\mathrm{Ab}$ levels in iPSC-derived neurons, suggesting that this approach could be translated to clinical trials [108].

Israel $e t$ al. demonstrated that neurons derived from iPSCs of both sporadic and familial forms of AD behave differently, with not only anomalous Ab expression but also aberrant expression of the aGSK-3b and hyperphosphorylated TAU forms, linking both Ab and TAU pathological hallmarks in iPSC models. In addition, treatment with b-secretase inhibitors led to a reduction in phosphorylated TAU and aGSK-3b levels [109]. TAU pathology is present in $\mathrm{AD}$ and other dementias, and iPSC-derived neurons with mutations in the MAPT gene showed altered TAU isoform expression, hyperphosphorylated TAU aggregates as well as different phenotypes associated with neurodegeneration [110]. Recently, we generated an iPSC-derived mutant TAU model that shows several phenotypes 
associated with neurodegeneration, among them the accumulation of pathogenic TAU. The characteristics of this iPSC-derived model allow the use of this platform for drug screening purposes [36].

As mentioned before, $\mathrm{AD}$ is a noncell autonomous disease, with involvement of several neural cell types. In fact, iPSC-derived astrocytes show pathological phenotypes in those derived from AD patients in comparison with those derived from healthy subjects [62,63], although still this platform has not been translated into the drug discovery field. As mentioned earlier, only recently specific protocols for the generation of iPSC-derived microglial cells have been developed [83-86], which have impeded modeling the role that microglia has in AD pathology. iPSC-derived microglia express main immunological risk variants associated to sporadic $\mathrm{AD}$ and are activated after $\mathrm{Ab}$ treatment [86]. Moreover, iPSC-derived microglia are starting to be used as a model where to test the effect of genetic variants in the microglial functionality [111,112], which can lead to the discovery of novel disease-mediated mechanisms. Finally, due to the involvement of several neural cell types in AD pathology, few studies have reported the generation of $3 \mathrm{D}$ organoid cultures that do not only reproduce $\mathrm{AD}$ pathology, but are also sensitive to drugs reverting disease phenotype $[92,113]$. Nonetheless, these approaches are far for being translated to HTS for drug discovery.

\section{Parkinson's disease}

PD symptoms are caused by a specific loss of DA neurons in the ventral midbrain [114]. Due to this localized neuron loss, important efforts have been made to generate and evaluate the correct neural population to be transplanted into the putamen of assessed models. The use of neural cells from human origin for therapeutic purposes in terms of promoting recovery after injury or for cell replacement purposes has been long tested in PD, due to the specific neuronal population and region to be targeted. Already in the early 1980s, researchers demonstrated that transplantation of fetal DA neurons successfully engrafted and restored functionality in a PD rat model [115]. This and other studies paved the way for the translation of this approach to patients 10-years later, when fetal DA neurons were successfully transplanted in a severe PD case, which engrafted and improved motor functions [116].

Since then, numerous studies have been performed employing neural cells from different origins [117]. Among them, the transplantation of DA neurons derived from iPSCs is taking shape to become a therapeutic possibility for PD patients. In this sense, recent protocols for obtaining DA neurons from hPSCs have notably improved the efficiency of obtaining these cells [118]. Preclinical studies have demonstrated that generated DA neurons, after purification, are successfully engrafted in a PD rat model [49]. The clinical suitability of hPSC-derived DA neurons has been further demonstrated, since these cells were transplanted in nonhuman primates treated with the neurotoxin 1-methyl-4-phenyl-1,2,3,6-tetrahydropyridine (MPTP), engrafting and improving motor function, surviving for at least 2 years without evidences of tumor formation [51]. This therapeutic approach is currently being translated to PD patients in a Phase I clinical trial.

Several groups have generated DA from iPSCs of PD patients and healthy controls and compared altered pathways between them with the intention of use these cells for disease modeling (reviewed in [118]). When compared with healthy controls or PD patients from different genetic backgrounds, different altered pathways were identified, with some of them susceptible of therapeutic modulation.

Hartfield et al. described the generation of mature and physiologically active DA neurons, which not only formed functional synapses but were susceptible to the DA-specific toxin 1-methyl-4-phenylpyridinium $\left(\mathrm{MPP}^{+}\right)$ that reduced mitochondrial membrane potential and altered mitochondrial morphology, suggesting the usefulness of this system for the testing of drugs [119]. Moreover, iPSC-derived DA neurons from PD patients with familial forms of the disease are characterized for presenting several pathogenic phenotypes, suggesting that a combination therapy may be necessary for PD, employing patient-derived cells for its elucidation [120]. Therefore, for the development of effective therapies for PD, iPSC-derived platforms seem suitable to test candidate drugs, which will most likely result in a combination of cell and drug therapy for the treatment of PD symptoms and disease course.

\section{Huntington's disease}

Huntington's disease (HD) is an inherited neurodegenerative disorder caused by an expanded stretch of CAG trinucleotide repeats in the huntingtin gene that results in the loss of GABAergic neurons in the striatum [121].

iPSC lines from HD patients have been generated that after differentiation toward NSCs or neurons exhibited decreased cell adhesion and ATP production, increased apoptosis and cell death after prolonged culture or BDNF withdrawal, and increased vulnerability to stressors. Moreover, the severity of these disease-associated phenotypes are directly influenced by the extension of CAG repeats [122]. These phenotypes can be reversed after specific 
CRISPR-Cas9-mediated gene correction [123], suggesting that those isogenic cell lines are particularly suitable for screening of drugs or identifying mechanisms that target phenotypes caused by the disease mutation [124].

Among the different phenotypes to be tested, aggregation of the mutated huntingtin protein has been the main readout employed for the search of effective drugs [125]. Another important phenotypes tested have been cell death and neuronal toxicity, as in HD, neuronal cell loss is observed in the cortex and striatum of patients [124]. Using this approach, several effective compounds have been identified, which need to be tested in more relevant HD models before translation to clinical trials for addressing their effectiveness to counteract HD [126]. In this sense, Nekrasov et al. reported in HD-derived neurons mutant huntingtin protein aggregation, increased number of lysosomes and autophagosomes and enhanced neuronal death during cell aging, with part of phenotypic reversal when cells were treated with a quinazoline derivative, EVP4593 [127]. In conclusion, iPSC-derived cells represent a more reliable platform where to perform screenings for finding drugs able to counteract pathogenic mechanisms associated to HD.

\section{Amyotrophic lateral sclerosis}

ALS is a fatal disease characterized by the degeneration of both upper and lower MNs that lead to death after 3-5 years of diagnosis [128]. Due to its devastating consequences and the lack of effective therapies counteracting effectively the disease progression, considerable actions are being taken for the development of effective therapies using different approaches. As mentioned before, several protocols have been developed for the specific differentiation of hPSCs into functional MNs $[53,54,56,57,129,130]$.

Several groups have used iPSC-derived MNs as a drug screening platform using cells derived from familial forms of the disease with mutations in TDP-43 [131,132], in the COORF72 gene [133] and in the SOD1 gene [37], which are the most common genes associated with familial ALS [134]. Among the different phenotypes encountered using iPSC-derived cells as a platform, several studies point out toward a disrupted membrane excitability in MNs derived from different ALS patients, suggesting that the development of effective drugs counteracting these phenotypes may have an important clinical relevance.

Burkhardt et al. generated iPSC-derived MNs from controls and sporadic ALS cases, finding that MNs derived from patients showed TDP-43 aggregates that recapitulate the pathology in post mortem tissue from one of the donor patients. Afterward, they established a high-content chemical screen measuring TDP-43 aggregation in MN-like cells, identifying an US FDA-approved small molecule (digoxin) showing efficacy [132]. Working with familial ALS cases with mutations in the SOD1 gene, Kiskinis et al. developed a high-throughput functional characterization of iPSC-derived MNs using optogenetic techniques and the employment of isogenic genetically corrected iPSC lines, highlighting the suitability of this system as a simple readout to evaluate the effect of candidate drugs on disease phenotypes [135].

In addition, it is worthy considering that ALS is not a cell-autonomous disease, as other neural cell types as astrocytes [136] or OLs [137] contribute to disease pathology, so they should be considered as well for the development of reliable drug testing platforms.

\section{MS \& other myelin-deficient diseases}

MS is an immune-mediated disease that leads to neuroinflammation, demyelination and neurodegeneration, leading to important and diverse functional deficits depending on the brain area affected [138]. In MS and other myelin-deficient diseases as leukodystrophies (group of congenital diseases where the structure and/or function of myelin is defective due mostly to mutations in myelin-related genes), one of the therapeutic strategies has been to develop specific drugs able to increase the myelin production by OLs within the CNS. Since this is a relatively easy phenomenon to be monitored, several approaches have been made to develop a reliable platform for drug screening purposes [139]. An important number of studies have developed myelinating platforms employing rodent primary OLs in combination with artificial homogeneous scaffolds [140] or presence of neurons [141]. Deshmukh et al. developed a HTS platform using primary rat optic nerve-derived OPCs, where they tested the effect of over 100,000 molecules on maturing these OPCs toward $\mathrm{MBP}^{+}$OLs. As a result of this HTS, they found several molecules that significantly improved the maturation of OPCs, but most of them were not adequate for their potential clinical uses as they had off-target activities, toxicity, poor brain exposure and/or demonstrated lack of in vivo efficacy. Therefore, the researchers focused on benztropine, a compound with a low effective concentration which is FDA-approved, orally available and crosses the blood-brain barrier, finding that benztropine improved remyelination in the experimental autoimmune encephalomyelitis model of MS [142]. 
Mei et al. developed a HTS-compatible drug screening system based on the capacity of primary murine OLs to surround and ensheath synthetic micropillars. Based on the expression of PDGFRa or MBP proteins, the authors tested the effect of 1000 bioactive compounds in the promotion of OPC (PDGFRa) or mature OLs (MBP), identifying a cluster of antimuscarinic compounds that enhance OL differentiation and remyelination, being the most efficacious compound clemastine [143], a widely available first-generation antihistamine, used for the treatment of seasonal allergies. Promotion of OL maturation induced by clemastine treatment showed beneficial in the cuprizone mouse model of demyelination [144] and for the treatment of hypoxic brain injury [145]. These evidences have led to the development of a crossover clinical trial where the researchers have evaluated the effect of clemastine on chronic MS patients, which showed efficacy in reducing delay in visual-evoked potentials [146].

Recently, in vitro myelination systems formed by OLs derived from murine ESCs have been developed [147], which are compatible with HTS platforms as well. Najm et al. employed mESC-derived OLs to constitute a drug screening platform. They tested over $700 \mathrm{FDA}$-approved drugs in their primary screen, which led to the identification of seven drugs that function at nanomolar doses selectively to enhance the generation of mature OLs $\left(\mathrm{MBP}^{+}\right)$from progenitor cells in vitro. Among them, two drugs, miconazole and clobetasol, enhanced myelination in ex vivo and in vivo models. Moreover, both drugs, when administered at the peak of the pathology in experimental autoimmune encephalomyelitis models, lead to reversal of disease severity, suggesting that they could be used as therapeutics for the treatment of MS or other de-/dysmyelinating diseases [148]. In a continuation work, this group studied the mechanism of action of miconazole and other previously identified drugs promoting myelin production, finding that all those drugs blocked an enzyme called CYP51, encouraging stem cells to form new OLs. CYP51 is involved in cholesterol synthesis, and the boost in OL production appeared to be due to the accumulation of the cholesterol precursors 8,9-unsaturated sterols that, per se, leads to an increased OL maturation. Using OLs derived from primary tissue and hPSC-derived organoids, researchers newly screened over 3000 drugs for their ability to mature OLs, finding that the top ten all caused a buildup of 8,9-unsaturated sterols, having found a druggable therapeutic target for the treatment of MS [149].

These studies indicate that the development of myelinating platforms for drug screenings can lead to novel discoveries susceptible to be translated to the clinical practice, which would benefit from the existence of more human-relevant drug testing platforms. In this sense, we have recently developed a methodology for an efficient generation of a myelinating platform formed by all human PSC-derived neurons and OLs, which is compatible with a HTS setting where the effect of candidate myelinating compounds can be evaluated [76]. Recently, a 3D myelinating system has been generated, where validation of candidate drugs could be performed in a human model close to in vivo situation [94].

Since it is believed that restoring myelin integrity could, at least partially, reverse the clinical symptoms, cell transplantation strategies have been as well tested for MS and other myelin-deficient diseases. In initial studies, researchers used human fetal tissue from where they isolated functional OLs. In 1987, Gumpel et al. generated cell homogenates from human fetal brain and transplanted these cells into the myelin-deficient shiverer mice, resulting in an effective myelination [150]. Recently, several studies have described specific OL/OPC isolation from human tissue, both from fetal [151] and adult origin [152,153]. However, the limited access to human tissue, the limited expansion capacity of these cells ex vivo, together with cell heterogeneity, make these cells not desirable for clinical uses. Human tissue-derived neural stem cells (NSCs) have also been used to study remyelination and recovery in several mouse models. Brustle et al. demonstrated that NSCs derived from human tissue are able to engraft, migrate, differentiate to OLs and promote myelination after transplantation in rats [154]. Since then, different studies have reported that human tissue-derived NSCs are able to differentiate, myelinate and improve functioning in different animal models of dys-/demyelination [155-157]. In spite of the positive results obtained when somatic neural cells are transplanted in animal models of different neurodegenerative conditions, the limited access to the tissue, the poor yield in terms of cell number and the great heterogeneity among samples remains a major hurdle for the use of these cells in the clinical setting, so alternative sources for the procurement of OLs are highly desirable.

\section{Conclusion}

The discovery and development of hPSC technology, especially when combined with gene-editing strategies, represents a novel and more reliable platform helpful for neurodegenerative disease modeling, a system that can be employed as well for performing screenings to identify new therapies able to counteract disease course of these devastating pathologies. 


\section{Future perspective}

Modeling of neurodegenerative diseases is a difficult task due to their complex and poorly elucidated pathology and the scarce access to human primary samples where to test hypothesis and candidate drugs. For these reasons, the development of hPSCs has revolutionized the field, though its technological progress has been slowed down due to the lack of efficient and robust protocols for the generation of specific disease-relevant neural cell types. Nonetheless, recent advances in the development of better protocols and the current existence of several iPSC banks from patients of selected neurodegenerative diseases with different genetic backgrounds should allow an extensive implementation of hPSC technology for disease modeling and drug testing efforts, which, most probably, will derive in better success rates of novel and effective therapies.

\section{Executive summary}

- Neurodegenerative diseases are complex pathological conditions and the elucidation of the underlying mechanisms has been precluded by the scarce access to human tissue and the lack of reliable models.

- Human-induced pluripotent stem cells (hiPSCs) represent an impactful technology for the generation of patient-specific relevant cell types to the disease.

- The development of effective gene-editing strategies as CRISPR-Cas9 together with iPSCs technology is allowing the elucidation of pathogenic pathways governing neurodegeneration.

- Since the discovery of iPSCs, specific and efficient protocols are being developed for the generation of the different disease-relevant neural cell types.

- The lack of efficiency and robustness for the derivation of iPSC-derived neural cell types has delayed the implementation of this technology as drug screening/validation platforms.

- In the past few years, iPSC-derived neural cells have started to be used as a platform where to test the effect of candidate drugs to counteract pathogenic mechanisms associated with neurodegenerative diseases.

- A review of the leading studies using iPSC-derived neural cells for disease modeling and drug screening platforms is provided for the main neurodegenerative conditions: Alzheimer's disease, Parkinson's disease, Huntington's disease, amyotrophic lateral sclerosis and multiple sclerosis and myelin-deficient diseases.

Financial \& competing interests disclosure

This work was supported by Instituto de Salud Carlos III (ISCiii) of Spain, cofinanced by FEDER funds from European Union, through grants PI15/00796 (to A Gutierrez), PI18/01557 (to A Gutierrez), Pl15/00957 (to J Vitorica) and PI18/01556 (to J Vitorica), by Junta de Andalucia Proyecto de Excelencia CTS- 2035 (to J Vitorica and A Gutierrez), by Consejeria de Salud of Junta de Andalucia grant PI-0276-2018 (to JA Garcia-Leon) and by CIBERNED. JA Garcia-Leon held a postdoctoral contract from the I Research Plan Propio of the University of Malaga. The authors have no other relevant affiliations or financial involvement with any organization or entity with a financial interest in or financial conflict with the subject matter or materials discussed in the manuscript apart from those disclosed.

No writing assistance was utilized in the production of this manuscript.

\section{Open access}

This work is licensed under the Attribution-NonCommercial-NoDerivatives 4.0 Unported License. To view a copy of this license, visit http://creativecommons.org/licenses/by-nc-nd/4.0/

\section{References}

Papers of special note have been highlighted as: $\bullet$ of interest; $\bullet \bullet$ of considerable interest

1. Dawson TM, Golde TE, Lagier-Tourenne C. Animal models of neurodegenerative diseases. Nat. Neurosci. 21(10), 1370-1379 (2018).

2. Patrícia B, Pereira A. Impact and prevention of neurodegenerative diseases in society: Alzheimer and Parkinson. In: Neurodegenerative Diseases. SM Group Open Access eBooks, DE, USA (2017).

3. Winblad B, Amouyel P, Andrieu S et al. Defeating Alzheimer's disease and other dementias: a priority for European science and society. Lancet Neurol. 15(5), 455-532 (2016).

4. Arthur KC, Calvo A, Price TR, Geiger JT, Chiò A, Traynor BJ. Projected increase in amyotrophic lateral sclerosis from 2015 to 2040. Nat. Commun. 7, 1-6 (2016).

5. Benito-León J. Are the prevalence and incidence of multiple sclerosis changing? Neuroepidemiology 36(3), 148-149 (2011).

6. Dorsey ER, Bloem BR. The Parkinson pandemic - a call to action. JAMA Neurol. 75(1), 9 (2018). 
7. Coyle-Gilchrist ITS, Dick KM, Patterson K et al. Prevalence, characteristics, and survival of frontotemporal lobar degeneration syndromes. Neurology 86(18), 1736-1743 (2016).

8. Gitler AD, Dhillon P, Shorter J. Neurodegenerative disease: models, mechanisms, and a new hope. Dis. Model. Mech. 10(5), 499-502 (2017).

9. Thomson JA. Embryonic stem cell lines derived from human blastocysts. Science 282(5391), 1145-1147 (1998).

10. Takahashi K, Yamanaka S. Induction of pluripotent stem cells from mouse embryonic and adult fibroblast cultures by defined factors. Cell 126(4), 663-676 (2006).

11. Takahashi $\mathrm{K}$, Tanabe $\mathrm{K}$, Ohnuki $\mathrm{M}$ et al. Induction of pluripotent stem cells from adult human fibroblasts by defined factors. Cell 131(5), 861-872 (2007).

- Initial paper describing the generation of human-induced pluripotent stem cells (hiPSCs).

12. Cao L, Tan L, Jiang T, Zhu X-C, Yu J-T. Induced pluripotent stem cells for disease modeling and drug discovery in neurodegenerative diseases. Mol. Neurobiol. 52(1), 244-255 (2015).

13. Gonzalez-Munoz E, Arboleda-Estudillo Y, Otu HH, Cibelli JB. Histone chaperone ASF1A is required for maintenance of pluripotency and cellular reprogramming. Science 345(6198), 822-825 (2014).

14. Hou P, Li Y, Zhang X et al. Pluripotent stem cells induced from mouse somatic cells by small-molecule compounds. Science 341(6146), 651-654 (2013).

15. Schlaeger TM, Daheron L, Brickler TR et al. A comparison of non-integrating reprogramming methods. Nat. Biotechnol. 33(1), 58-63 (2015).

16. Hockemeyer D, Jaenisch R, Biology C. Induced pluripotent stem cells meet genome editing. Cell Stem Cell. 18(5), 573-586 (2017).

17. Chiba K, Hockemeyer D. Genome editing in human pluripotent stem cells using site-specific nucleases. Methods Mol. Biol. 1239, 267-280 (2015).

18. Rouet P, Smih F, Jasin M. Introduction of double-strand breaks into the genome of mouse cells by expression of a rare-cutting endonuclease. Mol. Cell. Biol. 14(12), 8096-8106 (1994).

19. Bibikova M, Golic M, Golic KG, Carroll D. Targeted chromosomal cleavage and mutagenesis in drosophila using zinc-finger nucleases. Genetics 161(3), 1169-1175 (2002).

20. Bibikova M, Beumer K, Trautman JK, Carroll D. Enhancing gene targeting with designed zinc finger nucleases. Science (80-.) 300(5620), 764 (2003).

21. Hockemeyer D, Soldner F, Beard C et al. Efficient targeting of expressed and silent genes in human ESCs and iPSCs using zinc-finger nucleases. Nat. Biotechnol. 27(9), 851-857 (2009).

22. Christian M, Cermak T, Doyle EL et al. Targeting DNA double-strand breaks with TAL effector nucleases. Genetics 186(2), 757-761 (2010).

23. Hockemeyer D, Wang H, Kiani S et al. Genetic engineering of human pluripotent cells using TALE nucleases. Nat. Biotechnol. 29(8), 731-734 (2011).

24. Jinek M, Chylinski K, Fonfara I, Hauer M, Doudna JA, Charpentier E. A programmable dual-RNA-guided DNA endonuclease in adaptive bacterial immunity. Science 337(6096), 816-821 (2012).

25. Cong L, Ran FA, Cox D et al. Multiplex genome engineering using CRISPR/Cas systems. Science 339(6121), 819-823 (2013).

-. Interesting manuscript describing the procedure of an easy implementation of the CRISPR-Cas9 technology for gene editing of mammalian cells.

26. Giacalone JC, Sharma TP, Burnight ER et al. CRISPR-Cas9-based genome editing of human induced pluripotent stem cells. In: Current Protocols in Stem Cell Biology. John Wiley \& Sons, Inc., Hoboken, NJ, USA, 5B.7.1-5B.7.22 (2018).

27. Nii T, Kohara H, Marumoto T, Sakuma T, Yamamoto T, Tani K. Single-cell-state culture of human pluripotent stem cells increases transfection efficiency. Biores. Open Access 5(1), 127-136 (2016).

28. Ordovás L, Boon R, Pistoni M et al. Efficient recombinase-mediated cassette exchange in hPSCs to study the hepatocyte lineage reveals AAVS1 locus-mediated transgene inhibition. Stem Cell Reports 5(5), 918-931 (2015).

29. Yusa K. Seamless genome editing in human pluripotent stem cells using custom endonuclease-based gene targeting and the piggyBac transposon. Nat. Protoc. 8(10), 2061-2078 (2013).

30. Guo W, Naujock M, Fumagalli L et al. HDAC6 inhibition reverses axonal transport defects in motor neurons derived from FUS-ALS patients. Nat. Commun. 8(1), 861 (2017).

- Interesting paper modeling amyotrophic lateral sclerosis using iPSC-derived motor neurons that identified a pathogenic pathway susceptible of therapeutic modulation.

31. Ortiz-Virumbrales M, Moreno CL, Kruglikov I et al. CRISPR/Cas9-correctable mutation-related molecular and physiological phenotypes in iPSC-derived Alzheimer's PSEN2 N141I neurons. Acta Neuropathol. Commun. 5(1), 77 (2017).

32. Oksanen M, Petersen AJ, Naumenko N et al. PSEN1 mutant iPSC-derived model reveals severe astrocyte pathology in Alzheimer's disease. Stem Cell Reports 9, 1885-1897 (2017). 
33. Sun J, Carlson-Stevermer J, Das U et al. CRISPR/Cas9 editing of APP C-terminus attenuates $\beta$-cleavage and promotes $\alpha$-cleavage. Nat. Commun.10, 53 (2019).

34. Wang C, Najm R, Xu Q et al. Gain of toxic apolipoprotein E4 effects in human iPSC-derived neurons is ameliorated by a small-molecule structure corrector. Nat. Med. 24(5), 647-657 (2018).

- Demostrates the suitability of iPSC-derived neurons from Alzheimer's disease patients that can be used for disease modeling and drug screening.

35. Monteys AM, Ebanks SA, Keiser MS, Davidson BL. CRISPR/Cas9 editing of the mutant huntingtin allele in vitro and in vivo. Mol. Ther. 25(1), 12-23 (2017).

36. García-León JA, Cabrera-Socorro A, Eggermont K et al. Generation of a human induced pluripotent stem cell-based model for tauopathies combining three microtubule-associated protein tau mutations which displays several phenotypes linked to neurodegeneration. Alzheimer's Dement. 14, 1261-1280 (2018).

- Generation of an iPSC-based model for tauopathies using CRISPR-Cas9.

37. Kiskinis E, Sandoe J, Williams LA et al. Pathways disrupted in human ALS motor neurons identified through genetic correction of mutant SOD1. Cell Stem Cell. 14(6), 781-795 (2014).

38. Soldner F, Stelzer Y, Shivalila CS et al. Parkinson-associated risk variant in distal enhancer of $\alpha$-synuclein modulates target gene expression. Nature 533(7601), 95-99 (2016).

39. Zhang X-H, Tee LY, Wang X-G, Huang Q-S, Yang S-H. Off-target effects in CRISPR/Cas9-mediated genome engineering. Mol. Ther Nucleic Acids 4, e264 (2015).

40. Doerr A. CRISPR off-targets: a reassessment. Nat. Methods. 15(4), 229-230 (2018).

41. Akcakaya P, Bobbin ML, Guo JA et al. In vivo CRISPR editing with no detectable genome-wide off-target mutations. Nature 561(7723), 416-419 (2018).

42. De Angelis MT, Parrotta EI, Santamaria G, Cuda G. Short-term retinoic acid treatment sustains pluripotency and suppresses differentiation of human induced pluripotent stem cells. Cell Death Dis. 9(1), 6 (2018).

43. Tropepe V, Hitoshi S, Sirard C, Mak TW, Rossant J, van der Kooy D. Direct neural fate specification from embryonic stem cells: a primitive mammalian neural stem cell stage acquired through a default mechanism. Neuron 30(1), 65-78 (2001).

44. Chambers SM, Fasano CA, Papapetrou EP, Tomishima M, Sadelain M, Studer L. Highly efficient neural conversion of human ES and iPS cells by dual inhibition of SMAD signaling. Nat. Biotechnol. 27(3), 275-280 (2009).

45. Shi Y, Kirwan P, Smith J, Robinson HPC, Livesey FJ. Human cerebral cortex development from pluripotent stem cells to functional excitatory synapses. Nat. Neurosci. 15(3), 477-486 (2012).

46. Bardy C, van den Hurk M, Eames $\mathrm{T}$ et al. Neuronal medium that supports basic synaptic functions and activity of human neurons in vitro. Proc. Natl Acad. Sci. 112(20), e2725-e2734 (2015).

47. Gunhanlar N, Shpak G, van der Kroeg M et al. A simplified protocol for differentiation of electrophysiologically mature neuronal networks from human induced pluripotent stem cells. Mol. Psychiatry 23(5), 1336-1344 (2018).

48. Nguyen HN, Byers B, Cord B et al. LRRK2 mutant iPSC-Derived DA neurons demonstrate increased susceptibility to oxidative stress. Cell Stem Cell. 8(3), 267-280 (2011).

49. Sundberg M, Bogetofte H, Lawson T et al. Improved cell therapy protocols for Parkinson's disease based on differentiation efficiency and safety of hESC-, hiPSC-, and non-human primate iPSC-derived dopaminergic neurons. Stem Cells 31(8), 1548-1562 (2013).

50. Borgs L, Peyre E, Alix P et al. Dopaminergic neurons differentiating from LRRK2 G2019S induced pluripotent stem cells show early neuritic branching defects. Sci. Rep. 6(1), 33377 (2016).

51. Kikuchi T, Morizane A, Doi D et al. Human iPS cell-derived dopaminergic neurons function in a primate Parkinson's disease model. Nature. 548(7669), 592-596 (2017).

-• Demonstration of the suitability of iPSC-derived dopaminergic neurons to be used in cell therapy for Parkinson's disease.

52. Renton AE, Chiò A, Traynor BJ. State of play in amyotrophic lateral sclerosis genetics. Nat. Neurosci. 17(1), 17-23 (2014).

53. Dimos JT, Rodolfa KT, Niakan KK et al. Induced pluripotent stem cells generated from patients with ALS can be differentiated into motor neurons. Science 321(5893), 1218-1221 (2008).

54. Ebert AD, Yu J, Rose FF et al. Induced pluripotent stem cells from a spinal muscular atrophy patient. Nature 457(7227), 277-280 (2009).

55. Hu B-Y, Zhang S-C. Differentiation of spinal motor neurons from pluripotent human stem cells. Nat. Protoc. 4(9), $1295-1304$ (2009).

56. Amoroso MW, Croft GF, Williams DJ et al. Accelerated high-yield generation of limb-innervating motor neurons from human stem cells. J. Neurosci. 33(2), 574-586 (2013).

57. Maury Y, Côme J, Piskorowski RA et al. Combinatorial analysis of developmental cues efficiently converts human pluripotent stem cells into multiple neuronal subtypes. Nat. Biotechnol. 33(1), 89-96 (2015).

58. Tao Y, Zhang S-C. Neural subtype specification from human pluripotent stem cells. Cell Stem Cell. 19(5), 573-586 (2016). 
59. Zhang Y, Sloan SA, Clarke LE et al. Purification and characterization of progenitor and mature human astrocytes reveals transcriptional and functional differences with mouse. Neuron 89(1), 37-53 (2016).

60. Krencik R, Weick JP, Liu Y, Zhang Z-J, Zhang S-C. Specification of transplantable astroglial subtypes from human pluripotent stem cells. Nat. Biotechnol. 29(6), 528-534 (2011).

61. Tcw J, Wang M, Pimenova AA et al. An efficient platform for astrocyte differentiation from human induced pluripotent stem cells. Stem Cell Rep. 9(2), 600-614 (2017).

62. Jones VC, Atkinson-Dell R, Verkhratsky A, Mohamet L. Aberrant iPSC-derived human astrocytes in Alzheimer's disease. Cell Death Dis. 8(3), e2696-e2696 (2017).

63. Oksanen M, Petersen AJ, Naumenko N et al. PSEN1 mutant iPSC-derived model reveals severe astrocyte pathology in Alzheimer's disease. Stem Cell Rep. 9, 1885-1897 (2017).

64. Zhao J, Davis MD, Martens YA et al. APOE $\varepsilon 4 / \varepsilon 4$ diminishes neurotrophic function of human iPSC-derived astrocytes. Hum. Mol. Genet. 26(14), 2690-2700 (2017).

65. García-León JA, Verfaillie CM. Stem cell-derived oligodendroglial cells for therapy in neurological diseases. Curr. Stem Cell Res. Ther. 11(7), 569-577 (2016).

66. Nistor GI, Totoiu MO, Haque N, Carpenter MK, Keirstead HS. Human embryonic stem cells differentiate into oligodendrocytes in high purity and myelinate after spinal cord transplantation. Glia 49(3), 385-396 (2005).

67. Izrael M, Zhang P, Kaufman R et al. Human oligodendrocytes derived from embryonic stem cells: effect of noggin on phenotypic differentiation in vitro and on myelination in vivo. Mol. Cell. Neurosci. 34(3), 310-323 (2007).

68. Hu B-Y, Du Z-W, Zhang S-C. Differentiation of human oligodendrocytes from pluripotent stem cells. Nat. Protoc. 4(11), 1614-1622 (2009).

69. Czepiel M, Boddeke E, Copray S. Human oligodendrocytes in remyelination research. Glia 63(4), 513-530 (2015).

70. Jang J, Kang H-C, Kim H-S et al. Induced pluripotent stem cell models from X-linked adrenoleukodystrophy patients. Ann. Neurol. 70(3), 402-409 (2011).

71. Ogawa S, Tokumoto Y, Miyake J, Nagamune T. Induction of oligodendrocyte differentiation from adult human fibroblast-derived induced pluripotent stem cells. Vitr. Cell. Dev. Biol. 47(7), 464-469 (2011).

72. Wang S, Bates J, Li X et al. Human iPSC-derived oligodendrocyte progenitor cells can myelinate and rescue a mouse model of congenital hypomyelination. Cell Stem Cell. 12(2), 252-264 (2013).

73. Douvaras $\mathrm{P}$, Wang J, Zimmer $\mathrm{M}$ et al. Efficient generation of myelinating oligodendrocytes from primary progressive multiple sclerosis patients by induced pluripotent stem cells. Stem Cell Rep. 3(2), 250-259 (2014).

74. Piao J, Major T, Auyeung G et al. Human embryonic stem cell-derived oligodendrocyte progenitors remyelinate the brain and rescue behavioral deficits following radiation. Cell Stem Cell. 16(2), 198-210 (2015).

75. Ehrlich M, Mozafari S, Glatza M et al. Rapid and efficient generation of oligodendrocytes from human induced pluripotent stem cells using transcription factors. Proc. Natl Acad. Sci. 114(11), e2243-e2252 (2017).

76. García-León JA, Kumar M, Boon R et al. SOX10 single transcription factor-based fast and efficient generation of oligodendrocytes from human pluripotent stem cells. Stem Cell Rep. 10(2), 655-672 (2018).

77. Ginhoux F, Lim S, Hoeffel G, Low D, Huber T. Origin and differentiation of microglia. Front. Cell. Neurosci. 7, 45 (2013).

78. Karlsson KR, Cowley S, Martinez FO, Shaw M, Minger SL, James W. Homogeneous monocytes and macrophages from human embryonic stem cells following coculture-free differentiation in M-CSF and IL-3. Exp. Hematol. 36(9), 1167-1175 (2008).

79. van Wilgenburg B, Browne C, Vowles J, Cowley SA. Efficient, long term production of monocyte-derived macrophages from human pluripotent stem cells under partly-defined and fully-defined conditions. PLoS ONE 8(8), e71098 (2013).

80. van Wilgenburg B, Moore MD, James WS, Cowley SA. The productive entry pathway of HIV-1 in macrophages is dependent on endocytosis through lipid rafts containing CD4. PLoS ONE 9(1), e86071 (2014).

81. Etemad S, Zamin RM, Ruitenberg MJ, Filgueira L. A novel in vitro human microglia model: characterization of human monocyte-derived microglia. J. Neurosci. Methods 209(1), 79-89 (2012).

82. Ohgidani M, Kato TA, Setoyama D et al. Direct induction of ramified microglia-like cells from human monocytes: dynamic microglial dysfunction in Nasu-Hakola disease. Sci. Rep. 4, 4957 (2014).

83. Muffat J, Li Y, Yuan B et al. Efficient derivation of microglia-like cells from human pluripotent stem cells. Nat. Med. 22(11), 1358-1367 (2016).

84. Pandya $\mathrm{H}$, Shen MJ, Ichikawa DM et al. Differentiation of human and murine induced pluripotent stem cells to microglia-like cells. Nat. Neurosci. 20(5), 753-759 (2017).

85. Douvaras P, Sun B, Wang M et al. Directed differentiation of human pluripotent stem cells to microglia. Stem Cell Rep. 8(6), 1516-1524 (2017). 
86. Abud EM, Ramirez RN, Martinez ES et al. iPSC-derived human microglia-like cells to study neurological diseases. Neuron 94(2), 278-293.e9 (2017).

87. Wang H. Modeling neurological diseases with human brain organoids. Front. Synaptic Neurosci. 10, 15 (2018).

88. Lancaster MA, Renner M, Martin C-A et al. Cerebral organoids model human brain development and microcephaly. Nature 501(7467), 373-379 (2013).

- First demonstration of the generation of hiPSC-derived brain organoids.

89. Mariani J, Coppola G, Zhang P et al. FOXG1-dependent dysregulation of GABA/glutamate neuron differentiation in autism spectrum disorders. Cell 162(2), 375-390 (2015).

90. Stachowiak EK, Benson CA, Narla ST et al. Cerebral organoids reveal early cortical maldevelopment in schizophrenia - computational anatomy and genomics, role of FGFR1. Transl. Psychiatry 7(11), 6 (2017).

91. Dang J, Tiwari SK, Lichinchi G et al. Zika virus depletes neural progenitors in human cerebral organoids through activation of the innate immune receptor TLR3. Cell Stem Cell. 19(2), 258-265 (2016).

92. Choi SH, Kim YH, Hebisch M et al. A three-dimensional human neural cell culture model of Alzheimer's disease. Nature 515(7526), 274-278 (2014).

- Modeling Alzheimer's disease in a 3D iPSC-derived model.

93. Raja WK, Mungenast AE, Lin Y-T et al. Self-organizing 3D human neural tissue derived from induced pluripotent stem cells recapitulate Alzheimer's disease phenotypes. PLoS ONE 11(9), e0161969 (2016).

94. Madhavan M, Nevin ZS, Shick HE et al. Induction of myelinating oligodendrocytes in human cortical spheroids. Nat. Methods 15(9), 700-706 (2018).

95. Varma H, Lo D, Stockwell B. High throughput screening for neurodegeneration and complex disease phenotypes. Comb. Chem. High Throughput Screen. 11(3), 238-248 (2008).

96. Karapetyan YE, Sferrazza GF, Zhou M et al. Unique drug screening approach for prion diseases identifies tacrolimus and astemizole as antiprion agents. Proc. Natl Acad. Sci. 110(17), 7044-7049 (2013).

97. Cayo MA, Mallanna SK, Di Furio F et al. A drug screen using human iPSC-derived hepatocyte-like cells reveals cardiac glycosides as a potential treatment for hypercholesterolemia. Stem Cell 20, 478-489.e5 (2017).

98. del Álamo JC, Lemons D, Serrano R et al. High throughput physiological screening of iPSC-derived cardiomyocytes for drug development. Biochim. Biophys. Acta 1863(7), 1717-1727 (2016).

99. Polanco JC, Li C, Bodea L-G, Martinez-Marmol R, Meunier FA, Götz J. Amyloid- $\beta$ and tau complexity - towards improved biomarkers and targeted therapies. Nat. Rev. Neurol. 14(1), 22-39 (2017).

100. Gutierrez A, Vitorica J. Toward a new concept of Alzheimer's disease models: a perspective from neuroinflammation. J. Alzheimer's Dis. 64(Suppl. 1), S329-S338 (2018).

101. Navarro V, Sanchez-Mejias E, Jimenez S et al. Microglia in Alzheimer's disease: activated, dysfunctional or degenerative. Front. Aging Neurosci. 10, 140 (2018).

102. Sanchez-Mejias E, Navarro V, Jimenez S et al. Soluble phospho-tau from Alzheimer's disease hippocampus drives microglial degeneration. Acta Neuropathol. 132(6), 897-916 (2016).

103. Yagi T, Ito D, Okada Y et al. Modeling familial Alzheimer's disease with induced pluripotent stem cells. Hum. Mol. Genet. 20(23), 4530-4539 (2011).

104. Yahata N, Asai M, Kitaoka S et al. Anti-A $\beta$ drug screening platform using human iPS cell-derived neurons for the treatment of Alzheimer's disease. PLoS ONE 6(9), e25788 (2011)

105. Xu X, Lei Y, Luo J et al. Prevention of $\beta$-amyloid induced toxicity in human iPS cell-derived neurons by inhibition of cyclin-dependent kinases and associated cell cycle events. Stem Cell Res. 10(2), 213-227 (2013).

106. Winblad B, Amouyel P, Andrieu S et al. Defeating Alzheimer's disease and other dementias: a priority for European science and society. Lancet Neurol. 15(5), 455-532 (2016).

107. Mertens J, Stüber K, Wunderlich P et al. APP processing in human pluripotent stem cell-derived neurons is resistant to NSAID-based $\gamma$-secretase modulation. Stem Cell Rep. 1(6), 491-498 (2013).

108. Kondo T, Imamura K, Funayama $\mathrm{M}$ et al. iPSC-based compound screening and in vitro trials identify a synergistic anti-amyloid $\beta$ combination for Alzheimer's disease. Cell Rep. 21(8), 2304-2312 (2017).

109. Israel MA, Yuan SH, Bardy C et al. Probing sporadic and familial Alzheimer's disease using induced pluripotent stem cells. Nature 482(7384), 216-220 (2012).

110. Iovino M, Agathou S, González-Rueda A et al. Early maturation and distinct tau pathology in induced pluripotent stem cell-derived neurons from patients with MAPT mutations. Brain 138(11), 3345-3359 (2015).

111. Garcia-Reitboeck P, Phillips A, Piers TM et al. Human induced pluripotent stem cell-derived microglia-like cells harboring TREM2 missense mutations show specific deficits in phagocytosis. Cell Rep. 24(9), 2300-2311 (2018). 
112. Brownjohn PW, Smith J, Solanki R et al. Functional studies of missense TREM2 mutations in human stem cell-derived microglia. Stem Cell Rep. 10, 1-14 (2018).

113. J Siney E, Kurbatskaya K, Chatterjee S, Prasannan P, Mudher A, Willaime-Morawek S. Modelling neurodegenerative diseases in vitro: recent advances in 3D iPSC technologies. AIMS Cell Tissue Eng. 2(1), 1-23 (2018).

114. Poewe W, Seppi K, Tanner CM et al. Parkinson disease. Nat. Rev. Dis. Prim. 3, 17013 (2017).

115. Perlow MJ, Freed WJ, Hoffer BJ, Seiger A, Olson L, Wyatt RJ. Brain grafts reduce motor abnormalities produced by destruction of nigrostriatal dopamine system. Science 204(4393), 643-647 (1979).

116. Lindvall O, Brundin P, Widner H et al. Grafts of fetal dopamine neurons survive and improve motor function in Parkinson's disease. Science 247(4942), 574-577 (1990).

117. Yasuhara T, Kameda M, Sasaki T, Tajiri N, Date I. Cell therapy for Parkinson's disease. Cell Transplant. 26(9), 1551-1559 (2017).

118. Li H, Jiang H, Zhang B, Feng J. Modeling Parkinson's disease using patient-specific induced pluripotent stem cells. J. Parkinsons Dis. 8(4), 479-493 (2018).

119. Hartfield EM, Yamasaki-Mann M, Ribeiro Fernandes HJ et al. Physiological characterisation of human iPS-derived dopaminergic neurons. PLoS ONE 9(2), e87388 (2014).

120. Woodard CM, Campos BA, Kuo S-H et al. iPSC-derived dopamine neurons reveal differences between monozygotic twins discordant for Parkinson's disease. Cell Rep. 9(4), 1173-1182 (2014).

121. Bates GP, Dorsey R, Gusella JF et al. Huntington disease. Nat. Rev. Dis. Prim. 1, 15005 (2015).

122. HD iPSC Consortium. Induced pluripotent stem cells from patients with Huntington's disease show CAG-repeat-expansion-associated phenotypes. Cell Stem Cell. 11(2), 264-278 (2012).

123. Xu X, Tay Y, Sim B et al. Reversal of phenotypic abnormalities by CRISPR/Cas9-mediated gene correction in huntington disease patient-derived induced pluripotent stem cells. Stem Cell Rep. 8(3), 619-633 (2017).

124. Zhang N, Bailus BJ, Ring KL, Ellerby LM. IPSC-based drug screening for Huntingtons disease. Brain Res. 1638, 42-56 (2016).

125. Zuccato C, Valenza M, Cattaneo E. Molecular mechanisms and potential therapeutical targets in Huntington's disease. Physiol. Rev. 90(3), 905-981 (2010).

126. Sarantos MR, Papanikolaou T, Ellerby LM, Hughes RE. Pizotifen activates ERK and provides neuroprotection in vitro and in vivo in models of Huntington's disease. J. Huntingtons. Dis. 1(2), 195-210 (2012).

127. Nekrasov ED, Vigont VA, Klyushnikov SA et al. Manifestation of Huntington's disease pathology in human induced pluripotent stem cell-derived neurons. Mol. Neurodegener. 11(1), 27 (2016).

128. Taylor JP, Brown RH, Cleveland DW. Decoding ALS: from genes to mechanism. Nature 539(7628), 197-206 (2016).

129. Hu B-Y, Zhang S-C. Differentiation of spinal motor neurons from pluripotent human stem cells. Nat. Protoc. 4(9), 1295-1304 (2009).

130. Sances S, Bruijn LI, Chandran S et al. Modeling ALS with motor neurons derived from human induced pluripotent stem cells. Nat. Neurosci. 19(4), 542-553 (2016).

131. Egawa N, Kitaoka S, Tsukita K et al. Drug screening for ALS using patient-specific induced pluripotent stem cells. Sci. Transl. Med. 4(145), 145ra104-145ra104 (2012).

132. Burkhardt MF, Martinez FJ, Wright $S$ et al. A cellular model for sporadic ALS using patient-derived induced pluripotent stem cells. Mol. Cell. Neurosci. 56, 355-364 (2013).

133. Sareen D, O'Rourke JG, Meera P et al. Targeting RNA foci in iPSC-derived motor neurons from ALS patients with a C9ORF72 repeat expansion. Sci. Transl. Med. 5(208), 208ra149 (2013).

134. Wainger BJ, Kiskinis E, Mellin C et al. Intrinsic membrane hyperexcitability of amyotrophic lateral sclerosis patient-derived motor neurons. Cell Rep. 7(1), 1-11 (2014).

135. Kiskinis E, Kralj JM, Zou P et al. All-optical electrophysiology for high-throughput functional characterization of a human iPSC-derived motor neuron model of ALS. Stem Cell Rep. 10(6), 1991-2004 (2018).

136. Madill M, McDonagh K, Ma J et al. Amyotrophic lateral sclerosis patient iPSC-derived astrocytes impair autophagy via non-cell autonomous mechanisms. Mol. Brain. 10(1), 22 (2017).

137. Ferraiuolo L, Meyer K, Sherwood TW et al. Oligodendrocytes contribute to motor neuron death in ALS via SOD1-dependent mechanism. Proc. Natl Acad. Sci. USA 113(42), e6496-e6505 (2016).

138. Thompson AJ, Baranzini SE, Geurts J, Hemmer B, Ciccarelli O. Multiple sclerosis. Lancet 391(10130), 1622-1636 (2018).

139. Bechler ME, Swire M, Ffrench-Constant C. Intrinsic and adaptive myelination-a sequential mechanism for smart wiring in the brain. Dev. Neurobiol. 78(2), 68-79 (2018).

140. Lee S, Leach MK, Redmond SA et al. A culture system to study oligodendrocyte myelination processes using engineered nanofibers. Nat. Methods 9(9), 917-922 (2012).

141. Lariosa-Willingham KD, Rosler ES, Tung JS, Dugas JC, Collins TL, Leonoudakis D. Development of a central nervous system axonal myelination assay for high throughput screening. BMC Neurosci. 17(1), 16 (2016). 
142. Deshmukh VA, Tardif V, Lyssiotis CA et al. A regenerative approach to the treatment of multiple sclerosis. Nature 502(7471), 327-332 (2013).

143. Mei F, Fancy SPJ, Shen Y-AA et al. Micropillar arrays as a high-throughput screening platform for therapeutics in multiple sclerosis. Nat. Med. 20(8), 954-960 (2014).

144. Li Z, He Y, Fan S, Sun B. Clemastine rescues behavioral changes and enhances remyelination in the cuprizone mouse model of demyelination. Neurosci. Bull. 31(5), 617-625 (2015).

145. Cree BAC, Niu J, Hoi KK et al. Clemastine rescues myelination defects and promotes functional recovery in hypoxic brain injury. Brain 141(1), 85-98 (2018).

146. Green AJ, Gelfand JM, Cree BA et al. Clemastine fumarate as a remyelinating therapy for multiple sclerosis (ReBUILD): a randomised, controlled, double-blind, crossover trial. Lancet 390(10111), 2481-2489 (2017).

147. Kerman BE, Kim HJ, Padmanabhan K et al. In vitro myelin formation using embryonic stem cells. Development 142(12), 2213-2225 (2015).

148. Najm FJ, Madhavan M, Zaremba A et al. Drug-based modulation of endogenous stem cells promotes functional remyelination in vivo. Nature 522(7555), 216-220 (2015).

149. Hubler Z, Allimuthu D, Bederman I et al. Accumulation of 8,9-unsaturated sterols drives oligodendrocyte formation and remyelination. Nature 560(7718), 372-376 (2018).

150. Gumpel M, Lachapelle F, Gansmuller A, Baulac M, Baron van Evercooren A, Baumann N. Transplantation of human embryonic oligodendrocytes into shiverer brain. Ann. NY Acad. Sci. 495, 71-85 (1987).

151. Roy NS, Wang S, Harrison-Restelli C et al. Identification, isolation, and promoter-defined separation of mitotic oligodendrocyte progenitor cells from the adult human subcortical white matter. J. Neurosci. 19(22), 9986-9995 (1999).

152. Miron VE, Jung CG, Kim HJ, Kennedy TE, Soliven B, Antel JP. FTY720 modulates human oligodendrocyte progenitor process extension and survival. Ann. Neurol. 63(1), 61-71 (2008).

153. Ruffini F, Arbour N, Blain M, Olivier A, Antel JP. Distinctive properties of human adult brain-derived myelin progenitor cells. Am. J. Pathol. 165(6), 2167-2175 (2004).

154. Brüstle O, Choudhary K, Karram K et al. Chimeric brains generated by intraventricular transplantation of fetal human brain cells into embryonic rats. Nat. Biotechnol. 16(11), 1040-1044 (1998).

155. Cummings BJ, Uchida N, Tamaki SJ et al. Human neural stem cells differentiate and promote locomotor recovery in spinal cord-injured mice. Proc. Natl. Acad. Sci. USA 102(39), 14069-14074 (2005).

156. Pluchino S, Gritti A, Blezer E et al. Human neural stem cells ameliorate autoimmune encephalomyelitis in non-human primates. Ann. Neurol. 66(3), 343-354 (2009).

157. Windrem MS, Schanz SJ, Guo M et al. Neonatal chimerization with human glial progenitor cells can both remyelinate and rescue the otherwise lethally hypomyelinated shiverer mouse. Cell Stem Cell. 2(6), 553-565 (2008). 